\title{
Sum rules for the exchange-correlation energy functional of the extended constrained-search theory: Application to checking the validity of the vorticity expansion approximation of the current-density functional theory
}

\author{
M. Higuchi \\ Department of Physics, Faculty of Science, Shinshu University, Matsumoto 390-8621, Japan \\ K. Higuchi \\ Graduate School of Advanced Sciences of Matter, Hiroshima University, Higashi-Hiroshima 739-8527, Japan
}

(Received 27 August 2009; published 12 April 2010)

\begin{abstract}
We present four kinds of sum rules for the exchange-correlation energy functional of the extended constrainedsearch theory. They are applicable even to the conventional density functional theory. As an application of these sum rules, we utilize them to check the validity of the vorticity expansion approximation (VEA) of the current-density functional theory (CDFT). The VEA formula fulfils three of them, though the local density approximation formula of the CDFT fulfills only one. The validity of the VEA formula is thus confirmed successfully from the viewpoint of the sum rules.
\end{abstract}

DOI: 10.1103/PhysRevA.81.042505

PACS number(s): 31.15.eg, 71.15.Mb

\section{INTRODUCTION}

The current-density functional theory (CDFT) [1,2] and its relativistic version, relativistic current- and spin-density functional theory (RCSDFT) [3,4], provide useful methods to calculate the ground-state properties of materials where the orbital current is induced. Actual calculations have been performed so far for solids [5,6], quantum dots [7], and open-shell atoms [8] by using the local density approximation (LDA) of the CDFT [1,2,9] or the so-called exact exchange functional [8] as the exchange-correlation (xc) energy functional. However, effects of the orbital current are not always well described by means of such approximate forms [5-8]. Thus, a more adequate approximate form of the xc energy functional is needed in the field of the CDFT or RCSDFT.

The approximate form of the xc energy functional has been devised mainly with two strategies. One is based on the coupling-constant expression for the xc energy functional $[9,10]$. The key of this strategy is to approximate the couplingconstant-averaged pair correlation function by some known one, for example, the pair correlation function of the homogeneous electron liquid applied by a uniform magnetic field. In accordance with this strategy, the CDFT version of the LDA, the average-density approximation (ADA), and the weighteddensity approximation (WDA) have been proposed so far [9].

Another strategy is to develop the approximate form by requiring it to satisfy exact relations (sum rules) that should be fulfilled by the xc energy functional. ${ }^{1}$ This strategy has been used in developing the generalized gradient approximation (GGA) [11-15] and the density-moment expansion method [16-21] of the conventional density functional theory (DFT) $[22,23]$. To be more specific, Levy's asymptotic bound [24], which is one of sum rules for the xc energy functional of

\footnotetext{
${ }^{1}$ These exact relations are regarded as sum rules for the xc energy functional and often called so, because the xc energy functional can be written in a form of the coupling-constant integration. In this article, we call these relations sum rules
}

the DFT, has played an important role in the development of the Perdew-Burke-Ernzerhof (PBE) functional [13-15]. This bound is not satisfied with the LDA of the conventional DFT because the short-range correlation term causes the logarithmic divergence in the high density limit. In order to let the PBE functional fulfill this bound, the additional term that expresses the effect of the density gradient was devised. In the density-moment expansion method, the sum rules that are derived by using the virial theorem and the coordinate scaling technique [16,25-28] are effectively employed to determine its approximation form.

Also in the CDFT, many kinds of sum rules have been derived so far along with the later strategy $[1,2,9,10,29,30]$. These include the set of sum rules that are derived by using the gauge invariance, the virial theorem, the coordinate scaling of electrons, the adiabatic connection, and so on $[1,2,9,10,29,30]$. Using such sum rules as constraints, we have recently proposed the vorticity expansion approximation (VEA) for the xc energy functional of the CDFT [31-33]. This approximate form is developed to fulfill the gauge invariance and nineteen sum rules. The VEA formula for a homogeneous system is in quite good agreement with the exchange and correlation energies [34] of the homogeneous electron liquid applied by a uniform magnetic field [31-33].

Sum rules are useful not only for developing the approximate form of the xc energy functional but also for evaluating it. For the purpose of checking the validity of the VEA formula, the CDFT version of Levy's asymptotic bounds has been derived [32]. It is confirmed that the VEA formula fulfils the bounds, whereas the LDA of the CDFT does not. We also showed that the VEA formula satisfies other sum rules that are not directly used in constructing the VEA formula [33].

As mentioned, sum rules for the xc energy functional are indispensable in developing and/or evaluating the approximate form, and therefore it is essential to come up with new kinds of sum rules. In this article, we present four kinds of sum rules for the xc energy functional of the extended constrained-search (ECS) theory [10,35-39]. We apply these sum rules to checking the validity of the VEA formula of the 
CDFT. Three of them are satisfied with the VEA formula, though only one is satisfied with the CDFT-LDA formula. This shows that the VEA formula would work better than the CDFT-LDA in actual calculations. Thus, the sum rules derived here are expected to be quite useful in evaluating and/or developing the approximate form of the xc energy functional. Furthermore, in this article, we check the validity of the VEA formula systematically by using a total of fifty-six sum rules that correspond to the modified or generalized version of original sum rules of the DFT. It is found that the VEA formula satisfies many more sum rules than the CDFT-LDA.

Organization of this article is as follows. Detailed derivation of four kinds of sum rules is shown in Sec. II. Application of these sum rules to checking the validity of the VEA formula is shown in Sec. III A. Systematic checks via a total of sixty sum rules are performed in Sec. III B. Some concluding remarks are given in Sec. IV.

\section{SUM RULES FOR THE EXCHANGE-CORRELATION ENERGY FUNCTIONAL}

In this section, we derive sum rules for the xc energy functional of the ECS theory. The exchange and correlation energy functionals of the ECS theory are defined by

$$
E_{x}[\rho, \mathbf{X}]=\langle\Phi[\rho, \mathbf{X}]|\hat{W}| \Phi[\rho, \mathbf{X}]\rangle-U[\rho]
$$

and

$$
\begin{aligned}
E_{c}[\rho, \mathbf{X}]= & \langle\Psi[\rho, \mathbf{X}]|\hat{T}+\hat{W}| \Psi[\rho, \mathbf{X}]\rangle \\
& -\langle\Phi[\rho, \mathbf{X}]|\hat{T}+\hat{W}| \Phi[\rho, \mathbf{X}]\rangle,
\end{aligned}
$$

respectively [37], where $\rho(\mathbf{r})$ is the electron density and $\mathbf{X}(\mathbf{r})$ is the arbitrary physical quantity that is chosen as a basic variable. $\hat{T}$ and $\hat{W}$ denote the kinetic energy and the electron-electron interaction energy operators, respectively. $\Psi[\rho, \mathbf{X}]$ is the minimizing wave function in the definition of the universal functional of the ECS theory, and $\Phi[\rho, \mathbf{X}]$ is the minimizing Slater determinant in the definition of the kinetic energy functional of the reference system [10,35-37]. $U[\rho]$ stands for the Hartree energy term. For the purpose of deriving a set of sum rules for $E_{x}[\rho, \mathbf{X}]$ and $E_{c}[\rho, \mathbf{X}]$, we consider the system where the electron-electron interaction is multiplied by $\alpha$. By using the ECS [10,35-37], the universal functional of this system is defined by

$$
\begin{aligned}
F_{\alpha}[\rho, \mathbf{X}] & =\operatorname{Min}_{\Psi \rightarrow(\rho, \mathbf{X})}\langle\Psi|\hat{T}+\alpha \hat{W}| \Psi\rangle \\
& =\left\langle\Psi_{\alpha}[\rho, \mathbf{X}]|\hat{T}+\alpha \hat{W}| \Psi_{\alpha}[\rho, \mathbf{X}]\right\rangle,
\end{aligned}
$$

where $\Psi_{\alpha}[\rho, \mathbf{X}]$ denotes the minimizing wave function. Transforming $\Psi_{\alpha}[\rho, \mathbf{X}]$ by the coordinate scaling of electrons such that $\mathbf{r}_{i} \rightarrow \lambda^{-1} \mathbf{r}_{i} \quad(\lambda>0)$, we get $\lambda^{3 N / 2} \Psi_{\alpha}[\rho, \mathbf{X}]\left(\lambda \mathbf{r}_{1}, \ldots, \lambda \mathbf{r}_{N}\right)$, where $\lambda^{3 N / 2}$ is a normalizing constant. This wave function is referred to as the scaled wave function and is denoted by $\Psi_{\alpha}^{\lambda}[\rho, \mathbf{X}]$, that is,

$$
\Psi_{\alpha}^{\lambda}[\rho, \mathbf{X}]\left(\mathbf{r}_{1}, \ldots, \mathbf{r}_{N}\right)=\lambda^{3 N / 2} \Psi_{\alpha}[\rho, \mathbf{X}]\left(\lambda \mathbf{r}_{1}, \ldots, \lambda \mathbf{r}_{N}\right) .
$$

Using this wave function, we define the scaled basic variables $\rho_{\lambda}(\mathbf{r})$ and $\mathbf{X}_{\lambda}(\mathbf{r})$ by

$$
\rho_{\lambda}(\mathbf{r})=\left\langle\Psi_{\alpha}^{\lambda}[\rho, \mathbf{X}]|\hat{\rho}(\mathbf{r})| \Psi_{\alpha}^{\lambda}[\rho, \mathbf{X}]\right\rangle
$$

and

$$
\mathbf{X}_{\lambda}(\mathbf{r})=\left\langle\Psi_{\alpha}^{\lambda}[\rho, \mathbf{X}]|\hat{X}(\mathbf{r})| \Psi_{\alpha}^{\lambda}[\rho, \mathbf{X}]\right\rangle
$$

respectively, where $\hat{\rho}(\mathbf{r})$ and $\hat{X}(\mathbf{r})$ denote the operators of basic variables. Similarly to Eq. (3), the universal functional for the system where the electron-electron interaction is multiplied by $\alpha \lambda$ is defined by

$$
\begin{aligned}
F_{\alpha \lambda}[\rho, \mathbf{X}] & =\operatorname{Min}_{\Psi \rightarrow(\rho, \mathbf{X})}\langle\Psi|\hat{T}+\alpha \lambda \hat{W}| \Psi\rangle \\
& =\left\langle\Psi_{\alpha \lambda}[\rho, \mathbf{X}]|\hat{T}+\alpha \lambda \hat{W}| \Psi_{\alpha \lambda}[\rho, \mathbf{X}]\right\rangle,
\end{aligned}
$$

where $\Psi_{\alpha \lambda}[\rho, \mathbf{X}]$ is the minimizing wave function. As shown in Appendix A, the following relation holds:

$$
\Psi_{\alpha \lambda}\left[\rho_{\lambda}, \mathbf{X}_{\lambda}\right]\left(\mathbf{r}_{1}, \ldots, \mathbf{r}_{N}\right)=\Psi_{\alpha}^{\lambda}[\rho, \mathbf{X}]\left(\mathbf{r}_{1}, \ldots, \mathbf{r}_{N}\right) .
$$

By substituting $\alpha=\lambda^{-1}$ into Eq. (8) and using Eq. (4), we get

$$
\begin{aligned}
\Psi\left[\rho_{\lambda}, \mathbf{X}_{\lambda}\right]\left(\mathbf{r}_{1}, \ldots, \mathbf{r}_{N}\right) & =\Psi_{\lambda^{-1}}^{\lambda}[\rho, \mathbf{X}]\left(\mathbf{r}_{1}, \ldots, \mathbf{r}_{N}\right) \\
& =\lambda^{3 N / 2} \Psi_{\lambda^{-1}}[\rho, \mathbf{X}]\left(\lambda \mathbf{r}_{1}, \ldots, \lambda \mathbf{r}_{N}\right) .
\end{aligned}
$$

On the other hand, $\Phi\left[\rho_{\lambda}, \mathbf{X}_{\lambda}\right]$ can be written by

$$
\Phi\left[\rho_{\lambda}, \mathbf{X}_{\lambda}\right]\left(\mathbf{r}_{1}, \ldots, \mathbf{r}_{N}\right)=\lambda^{3 N / 2} \Phi[\rho, \mathbf{X}]\left(\lambda \mathbf{r}_{1}, \ldots, \lambda \mathbf{r}_{N}\right) .
$$

The proof of Eq. (10) is given in Ref. [10]. Substitution of Eqs. (9) and (10) into Eq. (2) leads to the correlation energy functional for $\rho_{\lambda}(\mathbf{r})$ and $\mathbf{X}_{\lambda}(\mathbf{r})$,

$$
\begin{aligned}
E_{c}\left[\rho_{\lambda}, \mathbf{X}_{\lambda}\right]= & \lambda^{2}\left\{\left\langle\Psi_{\lambda^{-1}}[\rho, \mathbf{X}]|\hat{T}| \Psi_{\lambda^{-1}}[\rho, \mathbf{X}]\right\rangle\right. \\
& -\langle\Phi[\rho, \mathbf{X}]|\hat{T}| \Phi[\rho, \mathbf{X}]\rangle\} \\
& +\lambda\left\{\left\langle\Psi_{\lambda^{-1}}[\rho, \mathbf{X}]|\hat{W}| \Psi_{\lambda^{-1}}[\rho, \mathbf{X}]\right\rangle\right. \\
& -\langle\Phi[\rho, \mathbf{X}]|\hat{W}| \Phi[\rho, \mathbf{X}]\rangle\} .
\end{aligned}
$$

Since the DFT version of Eq. (11) has been derived in Ref. [40], Eq. (11) corresponds to the generalization of the original expression of the DFT to the ECS one. The upper and lower bounds of $E_{c}\left[\rho_{\lambda}, \mathbf{X}_{\lambda}\right]$ can be obtained from Eq. (11) in a way similar to that in Ref. [40], which is listed in Table I (entries 59 and 60).

In what follows, we derive the set of sum rules that are related to the differential of $E_{c}\left[\rho_{\lambda}, \mathbf{X}_{\lambda}\right]$ with respect to the scaling parameter. By differentiating Eq. (11) with respect to $\lambda$, we get

$$
\begin{aligned}
\frac{d E_{c}}{}\left[\rho_{\lambda}, \mathbf{X}_{\lambda}\right] & d \lambda \\
= & 2 \lambda\left\{\left\langle\Psi_{\lambda^{-1}}[\rho, \mathbf{X}]|\hat{T}| \Psi_{\lambda^{-1}}[\rho, \mathbf{X}]\right\rangle-\langle\Phi[\rho, \mathbf{X}]|\hat{T}| \Phi[\rho, \mathbf{X}]\rangle\right\} \\
& +\left\langle\Psi_{\lambda^{-1}}[\rho, \mathbf{X}]|\hat{W}| \Psi_{\lambda^{-1}}[\rho, \mathbf{X}]\right\rangle-\langle\Phi[\rho, \mathbf{X}]|\hat{W}| \Phi[\rho, \mathbf{X}]\rangle \\
& -\left\{\left\langle\frac{d}{d \alpha} \Psi_{\alpha}[\rho, \mathbf{X}]|\hat{T}+\alpha \hat{W}| \Psi_{\alpha}[\rho, \mathbf{X}]\right\rangle\right. \\
& \left.+\left\langle\Psi_{\alpha}[\rho, \mathbf{X}]|\hat{T}+\alpha \hat{W}| \frac{d}{d \alpha} \Psi_{\alpha}[\rho, \mathbf{X}]\right\rangle\right\}_{\alpha=\lambda^{-1}} .
\end{aligned}
$$

Let us show that the third term of Eq. (12) vanishes. Since $\Psi_{\alpha}[\rho, \mathbf{X}]$ is the minimizing wave function of Eq. (3), $\Psi_{\alpha}[\rho, \mathbf{X}]$ 
obeys the following equation:

$$
\begin{aligned}
& \left\{\hat{T}+\alpha \hat{W}+\int v_{\alpha}[\rho, \mathbf{X}](\mathbf{r}) \hat{\rho}(\mathbf{r}) d \mathbf{r}\right. \\
& \left.\quad+\int \mathbf{a}_{\alpha}[\rho, \mathbf{X}](\mathbf{r}) \cdot \hat{\mathbf{X}}(\mathbf{r}) d \mathbf{r}\right\}\left|\Psi_{\alpha}[\rho, \mathbf{X}]\right\rangle=E_{\alpha}\left|\Psi_{\alpha}[\rho, \mathbf{X}]\right\rangle,
\end{aligned}
$$

where $E_{\alpha}, v_{\alpha}[\rho, \mathbf{X}](\mathbf{r})$ and $\mathbf{a}_{\alpha}[\rho, \mathbf{X}](\mathbf{r})$ are the real Lagrange multipliers that correspond to constraints on $\Psi_{\alpha}[\rho, \mathbf{X}]$, that is, $\Psi_{\alpha}[\rho, \mathbf{X}]$ is normalized to unity and yields both $\rho(\mathbf{r})$ and $\mathbf{X}(\mathbf{r})$. By using Eq. (13) and the fact that $\Psi_{\alpha}[\rho, \mathbf{X}]$ is normalized to unity, we get

$$
\begin{aligned}
& \left\langle\frac{d}{d \alpha} \Psi_{\alpha}[\rho, \mathbf{X}]\right| \hat{T}+\alpha \hat{W}+\int v_{\alpha}(\mathbf{r}) \hat{\rho}(\mathbf{r}) d \mathbf{r} \\
& \quad+\int \mathbf{a}_{\alpha}(\mathbf{r}) \cdot \hat{\mathbf{X}}(\mathbf{r}) d \mathbf{r}\left|\Psi_{\alpha}[\rho, \mathbf{X}]\right\rangle+\left\langle\Psi_{\alpha}[\rho, \mathbf{X}]\right| \hat{T}+\alpha \hat{W} \\
& \quad+\int v_{\alpha}(\mathbf{r}) \hat{\rho}(\mathbf{r}) d \mathbf{r}+\int \mathbf{a}_{\alpha}(\mathbf{r}) \cdot \hat{\mathbf{X}}(\mathbf{r}) d \mathbf{r}\left|\frac{d}{d \alpha} \Psi_{\alpha}[\rho, \mathbf{X}]\right\rangle=0 .
\end{aligned}
$$

It should be noted that Eq. (14) implies the Hellmann-Feynman theorem. For convenience, we rewrite Eq. (14) as follows:

$$
\begin{aligned}
& \left\langle\frac{d}{d \alpha} \Psi_{\alpha}[\rho, \mathbf{X}]|\hat{T}+\alpha \hat{W}| \Psi_{\alpha}[\rho, \mathbf{X}]\right\rangle \\
& \quad+\left\langle\Psi_{\alpha}[\rho, \mathbf{X}]|\hat{T}+\alpha \hat{W}| \frac{d}{d \alpha} \Psi_{\alpha}[\rho, \mathbf{X}]\right\rangle \\
& \quad+\int v_{\alpha}[\rho, \mathbf{X}](\mathbf{r}) \frac{d}{d \alpha}\left\{\left\langle\Psi_{\alpha}[\rho, \mathbf{X}]|\hat{\rho}(\mathbf{r})| \Psi_{\alpha}[\rho, \mathbf{X}]\right\rangle\right\} d \mathbf{r} \\
& \quad+\int \mathbf{a}_{\alpha}[\rho, \mathbf{X}](\mathbf{r}) \cdot \frac{d}{d \alpha}\left\{\left\langle\Psi_{\alpha}[\rho, \mathbf{X}]|\hat{\mathbf{X}}(\mathbf{r})| \Psi_{\alpha}[\rho, \mathbf{X}]\right\rangle\right\} d \mathbf{r}=0 .
\end{aligned}
$$

Since $\Psi_{\alpha}[\rho, \mathbf{X}]$ yields both $\rho(\mathbf{r})$ and $\mathbf{X}(\mathbf{r})$ that are independent of $\alpha$, we have

$$
\begin{aligned}
& \left\langle\frac{d}{d \alpha} \Psi_{\alpha}[\rho, \mathbf{X}]|\hat{T}+\alpha \hat{W}| \Psi_{\alpha}[\rho, \mathbf{X}]\right\rangle \\
& \quad+\left\langle\Psi_{\alpha}[\rho, \mathbf{X}]|\hat{T}+\alpha \hat{W}| \frac{d}{d \alpha} \Psi_{\alpha}[\rho, \mathbf{X}]\right\rangle=0 .
\end{aligned}
$$

Equation (16) means that the third term of Eq. (12) vanishes. Therefore, Eq. (12) becomes

$$
\begin{aligned}
\frac{d E_{c}}{c}\left[\rho_{\lambda}, \mathbf{X}_{\lambda}\right] & d \lambda \\
= & 2 \lambda\left\{\left\langle\Psi_{\lambda^{-1}}[\rho, \mathbf{X}]|\hat{T}| \Psi_{\lambda^{-1}}[\rho, \mathbf{X}]\right\rangle-\langle\Phi[\rho, \mathbf{X}]|\hat{T}| \Phi[\rho, \mathbf{X}]\rangle\right\} \\
& +\left\langle\Psi_{\lambda^{-1}}[\rho, \mathbf{X}]|\hat{W}| \Psi_{\lambda^{-1}}[\rho, \mathbf{X}]\right\rangle-\langle\Phi[\rho, \mathbf{X}]|\hat{W}| \Phi[\rho, \mathbf{X}]\rangle .
\end{aligned}
$$

This expression for the differential of $E_{c}\left[\rho_{\lambda}, \mathbf{X}_{\lambda}\right]$ with respect to the scaling parameter has not yet been derived even in the DFT. Equation (17) seems to be useful because this equation is reduced to the virial theorem [10] by taking the limit $\lambda \rightarrow 1$. The proof is given in Appendix B.

By using Eqs. (11) and (17), the following two equations are obtained;

$$
\begin{aligned}
\frac{d E_{c}\left[\rho_{\lambda}, \mathbf{X}_{\lambda}\right]}{d \lambda}= & 2 \frac{E_{c}\left[\rho_{\lambda}, \mathbf{X}_{\lambda}\right]}{\lambda}-\left\langle\Psi_{\lambda^{-1}}[\rho, \mathbf{X}]|\hat{W}| \Psi_{\lambda^{-1}}[\rho, \mathbf{X}]\right\rangle \\
& +\langle\Phi[\rho, \mathbf{X}]|\hat{W}| \Phi[\rho, \mathbf{X}]\rangle \\
\frac{1}{\lambda} \frac{d E_{c}\left[\rho_{\lambda}, \mathbf{X}_{\lambda}\right]}{d \lambda}= & \frac{E_{c}\left[\rho_{\lambda}, \mathbf{X}_{\lambda}\right]}{\lambda^{2}}+\left\langle\Psi_{\lambda^{-1}}[\rho, \mathbf{X}]|\hat{T}| \Psi_{\lambda^{-1}}[\rho, \mathbf{X}]\right\rangle \\
& -\langle\Phi[\rho, \mathbf{X}]|\hat{T}| \Phi[\rho, \mathbf{X}]\rangle .
\end{aligned}
$$

Here, let us introduce the assumption that $\rho(\mathbf{r})$ and $\mathbf{X}(\mathbf{r})$ are noninteracting $v$-representable. Under this assumption, it can be shown that $\operatorname{Min}_{\Psi \rightarrow(\rho, \mathbf{X})}\langle\Psi|\hat{T}| \Psi\rangle$ is equal to $\langle\Phi[\rho, \mathbf{X}]|\hat{T}| \Phi[\rho, \mathbf{X}]\rangle[10]$. Namely, we have

$$
\Psi_{0}[\rho, \mathbf{X}]=\Phi[\rho, \mathbf{X}]
$$

By taking the limit $\lambda \rightarrow \infty$ in Eqs. (18) and (19) and using Eq. (20), we obtain two kinds of sum rules:

$$
\begin{aligned}
& \lim _{\lambda \rightarrow \infty} \frac{d E_{c}\left[\rho_{\lambda}, \mathbf{X}_{\lambda}\right]}{d \lambda}=2 \lim _{\lambda \rightarrow \infty} \frac{E_{c}\left[\rho_{\lambda}, \mathbf{X}_{\lambda}\right]}{\lambda}, \\
& \lim _{\lambda \rightarrow \infty} \frac{1}{\lambda} \frac{d E_{c}\left[\rho_{\lambda}, \mathbf{X}_{\lambda}\right]}{d \lambda}=\lim _{\lambda \rightarrow \infty} \frac{E_{c}\left[\rho_{\lambda}, \mathbf{X}_{\lambda}\right]}{\lambda^{2}} .
\end{aligned}
$$

It should be noted that Eq. (18) [or Eq. (19)] combined with Eq. (11) is of course reduced to the virial theorem when taking the limit $\lambda \rightarrow 1$. As mentioned, by taking the other limit $(\lambda \rightarrow$ $\infty$ ) in Eqs. (18) and (19), we obtain Eqs. (21) and (22) that are different from the virial theorem. Therefore, Eqs. (18), (19), and the virial theorem are related to each other in the sense that these three can be derived from the same set of equations.

Also, other kinds of sum rules can be obtained from Eqs. (18) and (19). Since the expectation value of $\hat{W}$ with respect to $\Psi_{\lambda^{-1}}[\rho, \mathbf{X}]$ is necessarily positive in Eq. (18), we get

$$
\frac{d E_{c}\left[\rho_{\lambda}, \mathbf{X}_{\lambda}\right]}{d \lambda} \leqslant 2 \frac{E_{c}\left[\rho_{\lambda}, \mathbf{X}_{\lambda}\right]}{\lambda}+U[\rho]+E_{X}[\rho, \mathbf{X}],
$$

where Eq. (1) is used. Furthermore, under the assumption that $\rho(\mathbf{r})$ and $\mathbf{X}(\mathbf{r})$ are noninteracting $v$ representable, $\langle\Phi[\rho, \mathbf{X}]|\hat{T}| \Phi[\rho, \mathbf{X}]\rangle$ in Eq. (19) is equal to $\operatorname{Min}_{\Psi \rightarrow(\rho, \mathbf{X})}\langle\Psi|\hat{T}| \Psi\rangle$ [10]. Since $\Psi_{\lambda^{-1}}[\rho, \mathbf{X}]$ is one of wave functions that yield $\rho(\mathbf{r})$ and $\mathbf{X}(\mathbf{r})$, the following inequality holds:

$$
\left\langle\Psi_{\lambda^{-1}}[\rho, \mathbf{X}]|\hat{T}| \Psi_{\lambda^{-1}}[\rho, \mathbf{X}]\right\rangle \geqslant\langle\Phi[\rho, \mathbf{X}]|\hat{T}| \Phi[\rho, \mathbf{X}]\rangle
$$

By using Eqs. (19) and (24), we obtain

$$
\frac{E_{c}\left[\rho_{\lambda}, \mathbf{X}_{\lambda}\right]}{\lambda} \leqslant \frac{d E_{c}\left[\rho_{\lambda}, \mathbf{X}_{\lambda}\right]}{d \lambda} .
$$

Equations (23) and (25) are regarded as sum rules that give the upper and lower bounds of $d E_{c}\left[\rho_{\lambda}, \mathbf{X}_{\lambda}\right] / d \lambda$, respectively.

Equations (21), (22), (23), and (25) can be utilized in evaluating and/or developing the approximate forms of $E_{x}[\rho, \mathbf{X}]$ and $E_{c}[\rho, \mathbf{X}]$. It should be noted that these equations are reduced to sum rules for the xc energy functional of the conventional DFT by replacing $E_{x}[\rho, \mathbf{X}]$ and $E_{c}\left[\rho_{\lambda}, \mathbf{X}_{\lambda}\right]$ with $E_{x}[\rho]$ and $E_{c}\left[\rho_{\lambda}\right]$, respectively. 


\section{APPLICATION OF SUM RULES TO THE VEA FORMULA}

One of methods for utilizing sum rules is to evaluate the approximate form of the xc energy functional via them. In this section, we evaluate the VEA formula of the CDFT [31-33] by using various kinds of sum rules including the four kinds of sum rules derived in the previous section. In Sec. III A, we check whether the present four sum rules are satisfied with the VEA formula. Furthermore, in Sec. III A, four kinds of sum rules are utilized in evaluating the commonly used xc energy functional of the conventional DFT, such as the LDA [41], the PBE-GGA [13], and Lee-Yang-Parr (LYP)-GGA formula [42]. In Sec. III B, we perform exhaustive checking of the VEA and CDFT-LDA formulas by using all known sum rules so as to stress the validity of the VEA formula. We confirm that the VEA formula fulfils many more sum rules than the CDFT-LDA formula.

\section{A. Checking the validity of the VEA formula by using four kinds of sum rules}

We apply Eqs. (21), (22), (23), and (25) to checking the validity of the VEA formula [31-33] of the CDFT. Since the CDFT corresponds to the case where the paramagnetic current density $\mathbf{j}_{p}(\mathbf{r})$ is chosen as $\mathbf{X}(\mathbf{r})$ in the ECS theory [35], sum rules for exchange and correlation energy functionals of the CDFT can be obtained by replacing $\mathbf{X}(\mathbf{r})$ with $\mathbf{j}_{p}(\mathbf{r})$.

The VEA formulas are given as the functional of $\rho(\mathbf{r})$ and the vorticity $\boldsymbol{v}(\mathbf{r})$ that is defined by

$$
\boldsymbol{v}(\mathbf{r})=\nabla \times\left\{\frac{\mathbf{j}_{p}(\mathbf{r})}{\rho(\mathbf{r})}\right\} .
$$

The resultant VEA formulas for the exchange and correlation energy functionals are given by

$$
\bar{E}_{x}[\rho, \boldsymbol{v}]=E_{x}[\rho]+\bar{D}_{x} \frac{\hbar^{2}}{a_{H}^{3} \varepsilon_{H}} \int|\boldsymbol{v}(\mathbf{r})|^{2} d \mathbf{r}
$$

and

$$
\bar{E}_{c}[\rho, \boldsymbol{v}]=E_{c}[\rho]+\frac{\bar{C}_{0} \hbar^{2}}{a_{H}^{3} \varepsilon_{H}} \int \frac{e^{-\bar{\alpha} a_{H}^{3} \rho(\mathbf{r})} \rho(\mathbf{r})^{3}}{\left[\rho(\mathbf{r})-\bar{\delta} / a_{H}^{3}\right]^{3}}|\boldsymbol{v}(\mathbf{r})|^{2} d \mathbf{r},
$$

respectively [31-33]. Here, $a_{H}$ is the Bohr radius, and $\varepsilon_{H}$ is the Rydberg constant. $E_{x}[\rho]$ and $E_{c}[\rho]$ denote the exchange and correlation energy functionals of the conventional DFT, respectively. The dimensionless parameters $\bar{D}_{x}, \bar{C}_{0}, \bar{\alpha}$, and $\bar{\delta}$ are $3.76 \times 10^{-4},-4.669 \times 10^{-4}, 0.653$, and $1.0 \times 10^{-30}$, respectively [31-33], which have been determined by utilizing the exchange and correlation energies of the homogeneous electron liquid applied by a uniform magnetic field [34]. These formulas are constructed so as to comply with the gauge invariance and nineteen sum rules that are derived from coordinate scaling of electrons [31-33]. Note that since Eqs. (27) and (28) depend on $\mathbf{j}_{p}(\mathbf{r})$ through $\boldsymbol{v}(\mathbf{r})$, they are also recognized as the functionals of $\rho(\mathbf{r})$ and $\mathbf{j}_{p}(\mathbf{r})$.
From Eqs. (5), (6), and (26), the scaled basic variables and vorticity are calculated as

$$
\begin{aligned}
\rho_{\lambda}(\mathbf{r}) & =\lambda^{3} \rho(\lambda \mathbf{r}), \\
\mathbf{j}_{p \lambda}(\mathbf{r}) & =\lambda^{4} \mathbf{j}_{p}(\lambda \mathbf{r}), \\
\boldsymbol{v}_{\lambda}(\mathbf{r}) & =\lambda^{2} \boldsymbol{v}(\lambda \mathbf{r}) .
\end{aligned}
$$

By using Eqs. (28)-(31), $\bar{E}_{c}\left[\rho_{\lambda}, \boldsymbol{v}_{\lambda}\right]$ is written as

$$
\begin{aligned}
& \bar{E}_{c}\left[\rho_{\lambda}, \boldsymbol{v}_{\lambda}\right] \\
& =E_{c}\left[\rho_{\lambda}\right]+\frac{\lambda^{10} \bar{C}_{0} \hbar^{2}}{a_{H}^{3} \varepsilon_{H}} \int \frac{e^{-\bar{\alpha} a_{H}^{3} \lambda^{3} \rho(\mathbf{r})} \rho(\mathbf{r})^{3}}{\left[\lambda^{3} \rho(\mathbf{r})-\bar{\delta} / a_{H}^{3}\right]^{3}}|\boldsymbol{v}(\mathbf{r})|^{2} d \mathbf{r},
\end{aligned}
$$

where we used the transformation of the integration variables. By substituting Eq. (32) into Eqs. (21), (22), and (25), we can easily confirm in a similar way to that of Ref. [32] that the VEA formula is exactly satisfied with these sum rules. Concerning Eq. (23), we need numerical calculations in order to check whether it is satisfied with the VEA. The results are summarized in Table I (entries 55-58).

For comparison, we also check the validity of the LDA formula of the CDFT [1,2,9]. The LDA formulas for the exchange and correlation energy functionals are given by

$$
\begin{aligned}
& \bar{E}_{x}^{\mathrm{LDA}}[\rho, \boldsymbol{v}]=\int \rho(\mathbf{r}) \varepsilon_{x}^{\mathrm{homo}}\left(r_{s}(\mathbf{r}), \eta(\mathbf{r})\right) d \mathbf{r}, \\
& \bar{E}_{c}^{\mathrm{LDA}}[\rho, \boldsymbol{v}]=\int \rho(\mathbf{r}) \varepsilon_{c}^{\mathrm{homo}}\left(r_{s}(\mathbf{r}), \eta(\mathbf{r})\right) d \mathbf{r},
\end{aligned}
$$

where $\varepsilon_{x}^{\text {homo }}\left(r_{s}, \eta\right)$ and $\varepsilon_{c}^{\text {homo }}\left(r_{s}, \eta\right)$ denote the exchange and correlation energies of a homogeneous electron liquid applied by a uniform magnetic field, respectively; $r_{s}$ and $\eta$ are the density parameter and the occupation factor of the lowest Landau subband, respectively. $\varepsilon_{x}^{\text {homo }}\left(r_{s}, \eta\right)$ and $\varepsilon_{c}^{\text {homo }}\left(r_{s}, \eta\right)$ have been calculated by Skudlarski and Vignale within the random phase approximation (RPA) [43]. We used their results in checking whether the LDA formulas satisfy Eqs. (21), (22), (23), and (25). ${ }^{2}$

It is confirmed that the LDA formula of the CDFT fulfils only Eq. (22) but not Eqs. (23) or (25). Meanwhile, the VEA formula fulfils these three sum rules, as mentioned previously. This means that the VEA formula is more reasonable than the LDA formula from the view point of sum rules satisfied.

We also check whether the LDA, PBE-GGA, and LYPGGA formula of the conventional DFT satisfy four kinds of sum rules. The results are summarized in Table II. Although the LDA formula of the DFT satisfies Eqs. (21) and (22), it is not straightforward to judge whether the formula satisfies Eqs. (23) and (25). Actual densities are needed for these judgments. The same is equally true for the PBE-GGA and LYP-GGA formula

\footnotetext{
${ }^{2}$ There are other LDA formulas that have been developed by interpolating between the xc energy of a homogeneous electron gas applied by the strong magnetic field and that in the zero-magneticfield limit [7,44-48]. In this article, we use the LDA formula proposed by Skudlarski and Vignale [43].
} 
TABLE I. Sum rules that the VEA and the CDFT-LDA satisfy. Several sum rules cannot be judged to determine whether they are fulfilled by the VEA and LDA formulas and are marked by a dash.

\begin{tabular}{|c|c|c|c|}
\hline No. & Sum rules & CDFT-LDA & VEA \\
\hline 1 & $E_{x}\left[\rho_{\lambda}, \mathbf{j}_{p \lambda}\right]=\lambda E_{x}\left[\rho, \mathbf{j}_{p}\right]$ & Yes & Yes \\
\hline 2 & $E_{c}\left[\rho_{\lambda}, \mathbf{j}_{p \lambda}\right] \geqslant \lambda E_{c}\left[\rho, \mathbf{j}_{p}\right], \quad \lambda \geqslant 1$ & Yes & Yes \\
\hline 3 & $E_{c}\left[\rho_{\lambda}, \mathbf{j}_{p \lambda}\right] \leqslant \lambda E_{c}\left[\rho, \mathbf{j}_{p}\right], \quad \lambda \leqslant 1$ & Yes & Yes \\
\hline 4 & $\lim _{\lambda \rightarrow \infty} E_{c}\left[\rho_{\lambda}^{x}, \mathbf{j}_{p \lambda}^{x}\right]=0$ & - & Yes \\
\hline 5 & $\lim _{\lambda \rightarrow \infty} \lambda E_{c}\left[\rho_{\lambda}^{x}, \mathbf{j}_{p \lambda}^{x}\right]=\mathrm{const}$ & - & Yes \\
\hline 6 & $\lim _{\lambda} E_{c}\left[\rho_{\lambda}^{x}, \mathbf{j}_{p \lambda}^{x}\right]=0$ & Yes & Yes \\
\hline 7 & $\lim _{\lambda \rightarrow 0} \lambda^{-1} E_{c}\left[\rho_{\lambda}^{x}, \mathbf{j}_{p \lambda}^{x}\right]=0$ & No & Yes \\
\hline 8 & $\lim _{\lambda \rightarrow 0} \lambda^{-2} E_{c}\left[\rho_{\lambda}^{x}, \mathbf{j}_{p \lambda}^{x}\right]=\mathrm{const}$ & No & Yes \\
\hline 9 & $\lim _{\lambda \rightarrow \infty} E_{c}\left[\rho_{\lambda \lambda}^{x y}, \mathbf{j}_{p \lambda \lambda}^{x y}\right]=0$ & - & Yes \\
\hline 10 & $\lim _{\lambda \rightarrow \infty}^{\lambda \rightarrow \infty} \lambda E_{c}\left[\rho_{\lambda \lambda}^{x y}, \mathbf{j}_{p \lambda \lambda}^{x y}\right]=\mathrm{const}$ & - & Yes \\
\hline 11 & $\lim _{\lambda \rightarrow 0}^{\wedge \rightarrow \infty} E_{c}\left[\rho_{\lambda \lambda}^{x y}, \mathbf{j}_{p \lambda \lambda}^{x y}\right]=0$ & Yes & Yes \\
\hline 12 & $\lim _{\lambda \rightarrow 0}^{\lambda \rightarrow 0} \lambda^{-1} E_{c}\left[\rho_{\lambda \lambda}^{x y}, \mathbf{j}_{p \lambda \lambda}^{x y}\right]=0$ & No & Yes \\
\hline 13 & $\lim _{\lambda \rightarrow 0}^{\lambda \rightarrow 0} \lambda^{-2} E_{c}\left[\rho_{\lambda \lambda}^{x y}, \mathbf{j}_{p \lambda \lambda}^{x y}\right]=$ const & No & Yes \\
\hline 14 & $\lim _{\lambda \rightarrow \infty} E_{c}\left[\rho_{\lambda \lambda \lambda^{-1}}^{x y z}, \mathbf{j}_{p \lambda \lambda \lambda^{-1}}^{x y z}\right]=0$ & - & Yes \\
\hline 15 & $\lim _{\lambda \rightarrow \infty} \lambda E_{c}\left[\rho_{\lambda \lambda \lambda^{-1}}^{x y z}, \mathbf{j}_{p \lambda \lambda \lambda^{-1}}^{x y z}\right]=0$ & - & Yes \\
\hline 16 & $\lim _{\lambda \rightarrow \infty} \lambda^{2} E_{c}\left[\rho_{\lambda \lambda \lambda^{-1}}^{x y z}, \mathbf{j}_{p \lambda \lambda \lambda^{-1}}^{x y z}\right]=$ const & - & Yes \\
\hline 17 & $\lim _{\lambda \rightarrow 0} E_{c}\left[\rho_{\lambda \lambda \lambda^{-1}}^{x y z}, \mathbf{j}_{p \lambda \lambda \lambda^{-1}}^{x y z}\right]=0$ & Yes & Yes \\
\hline 18 & $\lim _{\lambda \rightarrow 0} \lambda^{-1} E_{c}\left[\rho_{\lambda \lambda \lambda^{-1}}^{x y z}, \mathbf{j}_{p \lambda \lambda \lambda^{-1}}^{x y z}\right]=0$ & No & Yes \\
\hline 19 & $\lim _{\lambda \rightarrow 0} \lambda^{-2} E_{c}\left[\rho_{\lambda \lambda \lambda^{-1}}^{x y z}, \mathbf{j}_{p \lambda \lambda \lambda^{-1}}^{x y z}\right]=$ const & No & Yes \\
\hline 20 & $\lim _{\lambda \rightarrow \infty} E_{c}\left[\rho_{\lambda}^{z}, \mathbf{j}_{p \lambda}^{z}\right]=0$ & No & Yes \\
\hline 21 & $\lim _{\lambda \rightarrow \infty} \lambda E_{c}\left[\rho_{\lambda}^{z}, \mathbf{j}_{p \lambda}^{z}\right]=\mathrm{const}$ & No & Yes \\
\hline 22 & $\lim _{\lambda \rightarrow 0} E_{c}\left[\rho_{\lambda}^{z}, \mathbf{j}_{p \lambda}^{z}\right]=0$ & - & Yes \\
\hline 23 & $\lim _{\lambda \rightarrow 0}^{\lambda \rightarrow 0} \lambda^{-1} E_{c}\left[\rho_{\lambda}^{z}, \mathbf{j}_{p \lambda}^{z}\right]=0$ & - & Yes \\
\hline 24 & $\lim _{\lambda \rightarrow 0} \lambda^{-2} E_{c}\left[\rho_{\lambda}^{z}, \mathbf{j}_{p \lambda}^{z}\right]=\mathrm{const}$ & - & Yes \\
\hline 25 & $\lim _{\lambda \rightarrow \infty}^{\lambda \rightarrow 0} E_{c}\left[\rho_{\lambda \lambda}^{y z}, \mathbf{j}_{p \lambda \lambda}^{y z}\right]=0$ & No & Yes \\
\hline 26 & $\lim _{\lambda \rightarrow \infty}^{\lambda \rightarrow \infty} \lambda E_{c}\left[\rho_{\lambda \lambda}^{y z}, \mathbf{j}_{p \lambda \lambda}^{y z}\right]=\mathrm{const}$ & No & Yes \\
\hline 27 & $\lim _{\lambda \rightarrow 0} E_{c}\left[\rho_{\lambda \lambda}^{y z}, \mathbf{j}_{p \lambda \lambda}^{y z}\right]=0$ & - & Yes \\
\hline 28 & $\lim _{\lambda \rightarrow 0}^{\lambda \rightarrow 0} \lambda^{-1} E_{c}\left[\rho_{\lambda \lambda}^{y z}, \mathbf{j}_{p \lambda \lambda}^{y z}\right]=0$ & - & Yes \\
\hline 29 & $\lim _{\lambda \rightarrow 0}^{\lambda \rightarrow 0} \lambda^{-2} E_{c}\left[\rho_{\lambda \lambda}^{y z}, \mathbf{j}_{p \lambda \lambda}^{y z}\right]=\mathrm{const}$ & - & Yes \\
\hline 30 & $\lim _{\lambda \rightarrow \infty} E_{c}\left[\rho_{\lambda \lambda^{-1}}^{x y}, \mathbf{j}_{p \lambda \lambda}^{x y}\right]=0$ & No & Yes \\
\hline 31 & $\lim _{\lambda \rightarrow \infty} \lambda E_{c}\left[\rho_{\lambda \lambda^{-1}}^{x y}, \mathbf{j}_{p \lambda \lambda^{-1}}^{x y}\right]=0$ & No & Yes \\
\hline 32 & $\lim _{\lambda \rightarrow \infty} \lambda^{2} E_{c}\left[\rho_{\lambda \lambda^{-1}}^{x y}, \mathbf{j}_{p \lambda \lambda^{-1}}^{x y}\right]=\mathrm{const}$ & No & Yes \\
\hline 33 & $\lim _{\lambda \rightarrow 0}^{\lambda \rightarrow \infty} E_{c}\left[\rho_{\lambda \lambda^{-1}}^{x y}, \mathbf{j}_{p \lambda \lambda^{-1}}^{x y}\right]=0$ & No & No \\
\hline 34 & $\lim _{\lambda \rightarrow 0} \lambda^{-1} E_{c}\left[\rho_{\lambda \lambda^{-1}}^{x y}, \mathbf{j}_{p \lambda \lambda^{-1}}^{x y}\right]=0$ & No & No \\
\hline 35 & $\lim _{\lambda \rightarrow 0} \lambda^{-2} E_{c}\left[\rho_{\lambda \lambda^{-1}}^{x y}, \mathbf{j}_{p \lambda \lambda^{-1}}^{x y}\right]=$ const & No & No \\
\hline 36 & $\lim _{\lambda \rightarrow \infty} E_{c}\left[\rho_{\lambda^{-1} \lambda}^{x y}, \mathbf{j}_{p \lambda^{-1} \lambda}^{x y}\right]=0$ & No & No \\
\hline 37 & $\lim _{\lambda \rightarrow \infty}^{\lambda \rightarrow \infty} \lambda E_{c}\left[\rho_{\lambda^{-1} \lambda}^{x y}, \mathbf{j}_{p \lambda^{-1} \lambda}^{x y}\right]=0$ & No & No \\
\hline 38 & $\lim _{\lambda \rightarrow \infty} \lambda^{2} E_{c}\left[\rho_{\lambda^{-1} \lambda}^{x y}, \mathbf{j}_{p \lambda^{-1} \lambda}^{x y}\right]=$ const & No & No \\
\hline 39 & $\lim _{\lambda \rightarrow 0} E_{c}\left[\rho_{\lambda-1 \lambda}^{x y}, \mathbf{j}_{p \lambda^{-1} \lambda}^{x y}\right]=0$ & No & Yes \\
\hline 40 & $\lim _{\lambda \rightarrow 0} \lambda^{-1} E_{c}\left[\rho_{\lambda^{-1} \lambda}^{x y}, \mathbf{j}_{p \lambda^{-1} \lambda}^{x y}\right]=0$ & No & Yes \\
\hline 41 & $\lim _{\lambda \rightarrow 0} \lambda^{-2} E_{c}\left[\rho_{\lambda-1 \lambda}^{x y}, \mathbf{j}_{p \lambda^{-1} \lambda}^{x y}\right]=\mathrm{const}$ & No & Yes \\
\hline 42 & $\lim _{\lambda \rightarrow \infty} E_{c}\left[\rho_{\lambda \lambda^{-1}}^{y z}, \mathbf{j}_{p \lambda \lambda^{-1}}^{y z}\right]=0$ & No & No \\
\hline 43 & $\lim _{\lambda \rightarrow \infty} \lambda E_{c}\left[\rho_{\lambda \lambda^{-1}}^{y z}, \mathbf{j}_{p \lambda \lambda^{-1}}^{y z}\right]=0$ & No & No \\
\hline
\end{tabular}


TABLE I. (Continued.)

\begin{tabular}{|c|c|c|c|}
\hline No. & Sum rules & CDFT-LDA & VEA \\
\hline 44 & $\lim _{\lambda \rightarrow \infty} \lambda^{2} E_{c}\left[\rho_{\lambda \lambda^{-1}}^{y z}, \mathbf{j}_{p \lambda \lambda^{-1}}^{y z}\right]=$ const & No & No \\
\hline 45 & $\lim _{\lambda \rightarrow 0} E_{c}\left[\rho_{\lambda \lambda^{-1}}^{y z}, \mathbf{j}_{p \lambda \lambda^{-1}}^{y z}\right]=0$ & No & No \\
\hline 46 & $\lim _{\lambda \rightarrow 0} \lambda^{-1} E_{c}\left[\rho_{\lambda \lambda^{-1}}^{y z}, \mathbf{j}_{p \lambda \lambda^{-1}}^{y z}\right]=0$ & No & No \\
\hline 47 & $\lim _{\lambda \rightarrow 0} \lambda^{-2} E_{c}\left[\rho_{\lambda \lambda^{-1}}^{y z}, \mathbf{j}_{p \lambda \lambda^{-1}}^{y z}\right]=$ const & No & No \\
\hline 48 & 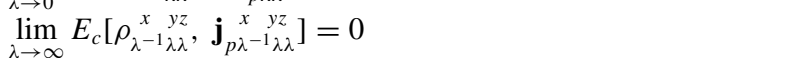 & No & Yes \\
\hline 49 & 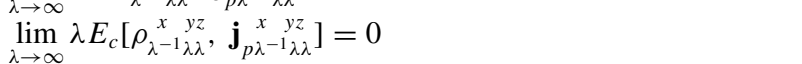 & No & Yes \\
\hline 50 & $\lim _{\lambda \rightarrow \infty} \lambda^{2} E_{c}\left[\rho_{\lambda^{-1} \lambda \lambda}^{x}, y z, \mathbf{j}_{p \lambda^{-1}{ }_{\lambda \lambda}^{x}}^{x}\right]=$ const & No & Yes \\
\hline 51 & $\lim _{\lambda \rightarrow 0} E_{c}\left[\begin{array}{ccc}x & y z \\
\lambda^{-1} \lambda \lambda & , \mathbf{j}_{p \lambda^{-1}}^{x} \quad y z\end{array}\right]=0$ & - & Yes \\
\hline 52 & 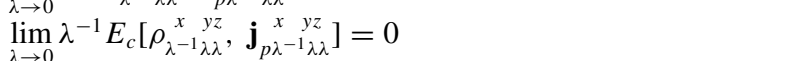 & - & Yes \\
\hline 53 & 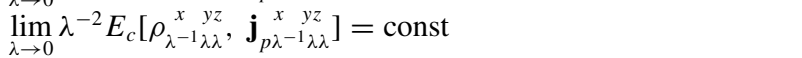 & - & Yes \\
\hline 54 & $\lim _{\lambda \rightarrow \infty} E_{c}\left[\rho_{\lambda}, \mathbf{j}_{p \lambda}\right]=$ const & No & Yes \\
\hline 55 & $\lim _{\lambda \rightarrow \infty} \frac{d E_{c}\left[\rho_{\lambda}, \mathbf{j}_{p \lambda}\right]}{d \lambda}=2 \lim _{\lambda \rightarrow \infty} \frac{E_{c}\left[\rho_{\lambda}, \mathbf{j}_{p \lambda}\right]}{\lambda}$ & No & Yes \\
\hline 56 & $\lim _{\lambda \rightarrow \infty} \frac{1}{\lambda} \frac{d E_{c}\left[\rho_{\lambda}, \mathbf{j}_{p \lambda}\right]}{d \lambda}=\lim _{\lambda \rightarrow \infty} \frac{E_{c}\left[\rho_{\lambda}, \mathbf{j}_{p \lambda}\right]}{\lambda^{2}}$ & Yes & Yes \\
\hline 57 & $\frac{d E_{c}\left[\rho_{\lambda}, \mathbf{j}_{p \lambda}\right]}{d \lambda} \leqslant 2 \frac{E_{c}\left[\rho_{\lambda}, \mathbf{j}_{p \lambda}\right]}{\lambda}+U[\rho]+E_{x}\left[\rho, \mathbf{j}_{p}\right]$ & - & - \\
\hline 58 & $\frac{E_{c}\left[\rho_{\lambda}, \mathbf{j}_{p \lambda}\right]}{\lambda} \leqslant \frac{d E_{c}\left[\rho_{\lambda}, \mathbf{j}_{p \lambda}\right]}{d \lambda}$ & No & Yes \\
\hline 59 & $-E_{x}\left[\rho, \mathbf{j}_{p}\right]-U[\rho] \leqslant \frac{E_{c}\left[\rho_{\lambda}, \mathbf{J}_{p \lambda}\right]}{\lambda}$ & - & Yes \\
\hline 60 & $\frac{E_{c}\left[\rho_{\lambda}, \mathbf{j}_{p \lambda}\right]}{\lambda} \leqslant(2-\lambda) E_{c}\left[\rho, \mathbf{j}_{p}\right]+(1-\lambda)\left[\frac{d E_{c}\left[\rho_{\zeta}, \mathbf{j}_{p \zeta}\right]}{d \zeta}\right]_{\zeta=1}$ & - & - \\
\hline
\end{tabular}

of the DFT. It would be meaningful to develop the xc energy functional that clearly satisfy not only Eqs. (21) and (22) but also Eqs. (23) and (25).

\section{B. Exhaustive checks of the VEA formula via a total of sixty sum rules}

In this section, we check the VEA and LDA formulas systematically by using a total of sixty sum rules. These sum rules have been derived so far by several authors $[1,2,9,10,29,30]$. The results are summarized in Table I. In checking the CDFT-LDA formula via sum rules 4 to 53, we assume that the magnetic field is parallel to the $x$ axis [9]. This assumption

TABLE II. Evaluation of the LDA, PBE, and LYP formulas of the conventional DFT by means of four kinds of sum rules. Concerning the latter two sum rules, we need actual densities to judge whether they satisfy the sum rules, and these are marked by a dash.

\begin{tabular}{lccc}
\hline \hline Sum rules & LDA & PBE & LYP \\
& {$[41]$} & {$[13]$} & {$[42]$} \\
$\lim _{\lambda \rightarrow \infty} \frac{d E_{c}\left[\rho_{\lambda}\right]}{d \lambda}=2 \lim _{\lambda \rightarrow \infty} \frac{E_{c}\left[\rho_{\lambda}\right]}{\lambda}$ & Yes & Yes & Yes \\
$\lim _{\lambda \rightarrow \infty} \frac{1}{\lambda} \frac{d E_{c}\left[\rho_{\lambda}\right]}{d \lambda}=\lim _{\lambda \rightarrow \infty} \frac{E_{c}\left[\rho_{\lambda}\right]}{\lambda^{2}}$ & Yes & Yes & Yes \\
$\frac{d E_{c}\left[\rho_{\lambda}\right]}{d \lambda} \leqslant 2 \frac{E_{c}\left[\rho_{\lambda}\right]}{\lambda}+U[\rho]+E_{x}[\rho]$ & - & - & - \\
$\frac{E_{c}\left[\rho_{\lambda}\right]}{\lambda} \leqslant \frac{d E_{c}\left[\rho_{\lambda}\right]}{d \lambda}$ & - & - & - \\
\hline \hline
\end{tabular}

is also utilized in checking the VEA formula via sum rules 30 to 47 . It should be noted that sum rules 59 and 60 , which were originally derived in the conventional DFT by Levy [40], are generalized to suitable ones for the VEA and LDA formulas of the CDFT. It is confirmed that $77 \%$ of sum rules are fulfilled by the VEA formula whereas only $12 \%$ of sum rules are fulfilled by the LDA formula. The VEA formula satisfies many more sum rules than the CDFT-LDA one. This trend is similar to that of the previous section. Thus, the VEA formula has wellbehaved form in comparison with the CDFT-LDA formula.

At the end of this section, we give a brief comment on twelve sum rules that are not satisfied by the VEA formula (33-38, 42-47). Since the sum rule generally gets rid of the difficulties that lead to unphysical results, we may expect that the more sum rules the VEA formula fulfils, the better it works for describing the ground-state properties of solids where the orbital current is induced. It seems that these sum rules provide a useful guideline for modifying the present VEA formula to more sophisticated one.

\section{CONCLUDING REMARKS}

We have derived four kinds of sum rules for the xc energy functional of the ECS theory by means of the coordinate scaling technique and the Hellmann-Feynman theorem. These sum rules can be utilized in developing, modifying, and evaluating the approximate form of the xc energy functional of the ECS theory. As shown in Sec. II, two of them [Eqs. (21) and (22)] can be related to the well-known virial theorem by 
taking a different limit of the scaling parameter. ${ }^{3}$ From this point, these sum rules especially seem to be useful for the above-mentioned aim.

As an application of these four sum rules, we have evaluated the VEA and LDA formulas of the CDFT by using them. The VEA formula is exactly satisfied by three of them. We cannot judge at the present stage whether the remaining one [Eq. (23)] is fulfilled by the VEA formula. This is because the actual profiles of the electron density and vorticity are indispensable for the check. On the other hand, the LDA formula of the CDFT is satisfied with only one of four sum rules. This tendency such that the VEA formula fulfils many more sum rules than the CDFT-LDA formula can also be seen for other checks using a total of sixty sum rules, which are shown in Table I. Thus, we can say that the VEA formula would be better behaved than the CDFT-LDA formula.

Finally, we would like to emphasize that the kinds of sum rules derived here might be useful also in the field of the conventional DFT, as mentioned in Sec. III A.

\section{ACKNOWLEDGMENTS}

This work was partially supported by a Grant-in-Aid for Scientific Research (No. 19540399) of the Ministry of Education, Culture, Sports, Science, and Technology, Japan.

\section{APPENDIX A: PROOF OF EQ. (8)}

In this appendix, we give the proof of Eq. (8). Since $\Psi_{\alpha}[\rho, \mathbf{X}]$ is defined as the minimizing wave function in Eq. (3), $\Psi_{\alpha}[\rho, \mathbf{X}]$ obeys the following equation:

$$
\begin{gathered}
\left\{\hat{T}+\alpha \hat{W}+\int v_{\alpha}[\rho, \mathbf{X}](\mathbf{r}) \hat{\rho}(\mathbf{r}) d \mathbf{r}+\int \mathbf{a}_{\alpha}[\rho, \mathbf{X}](\mathbf{r}) \cdot \hat{\mathbf{X}}(\mathbf{r}) d \mathbf{r}\right\} \\
\times \Psi_{\alpha}[\rho, \mathbf{X}]\left(\mathbf{r}_{1}, \ldots, \mathbf{r}_{N}\right)=E_{\alpha} \Psi_{\alpha}[\rho, \mathbf{X}]\left(\mathbf{r}_{1}, \ldots, \mathbf{r}_{N}\right),
\end{gathered}
$$

where $E_{\alpha}, v_{\alpha}[\rho, \mathbf{X}](\mathbf{r})$ and $\mathbf{a}_{\alpha}[\rho, \mathbf{X}](\mathbf{r})$ denote the Lagrange multipliers that correspond to constraints on $\Psi_{\alpha}[\rho, \mathbf{X}]$, that is, $\Psi_{\alpha}[\rho, \mathbf{X}]$ is normalized to unity and yields $\rho(\mathbf{r})$ and $\mathbf{X}(\mathbf{r})$. We transform Eq. (A1) by the coordinate scaling of electrons such that $\mathbf{r}_{i} \rightarrow \lambda^{-1} \mathbf{r}_{i}$. By the transformation, $\hat{T}, \hat{W}$, and $\hat{\rho}(\mathbf{r})$ are changed into $\hat{T} / \lambda^{2}, \hat{W} / \lambda$, and $\hat{\rho}(\mathbf{r} / \lambda) / \lambda^{3}$, respectively. Concerning $\hat{\mathbf{X}}(\mathbf{r})$, we assume that $\hat{\mathbf{X}}(\mathbf{r})$ is transformed into $\hat{\mathbf{X}}(\mathbf{r} / \lambda) / \lambda^{d}$, where $d$ is a real number. For the illustrative case where $\hat{\mathbf{X}}(\mathbf{r})$ corresponds to the paramagnetic current density, $d$ is shown to be 4 . By the coordinate scaling, Eq. (A1) is transformed into

$$
\begin{aligned}
& \left\{\hat{T}+\alpha \lambda \hat{W}+\int \lambda^{2} v_{\alpha}[\rho, \mathbf{X}](\lambda \mathbf{r}) \hat{\rho}(\mathbf{r}) d \mathbf{r}\right. \\
& \left.\quad+\int \lambda^{5-d} \mathbf{a}_{\alpha}[\rho, \mathbf{X}](\lambda \mathbf{r}) \cdot \hat{\mathbf{X}}(\mathbf{r}) d \mathbf{r}\right\} \\
& \quad \times \Psi_{\alpha}[\rho, \mathbf{X}]\left(\lambda \mathbf{r}_{1}, \ldots, \lambda \mathbf{r}_{N}\right)=\lambda^{2} E_{\alpha} \Psi_{\alpha}[\rho, \mathbf{X}]\left(\lambda \mathbf{r}_{1}, \ldots, \lambda \mathbf{r}_{N}\right) .
\end{aligned}
$$

\footnotetext{
${ }^{3}$ Of course, the derivation of the virial theorem via Eqs. (11) and (18) is not obvious and is different from the previous derivation [10]. Equation (18) itself is derived in this article.
}

With use of Eq. (4), Eq. (A2) becomes

$$
\begin{aligned}
\{\hat{T} & +\alpha \lambda \hat{W}+\int \lambda^{2} v_{\alpha}[\rho, \mathbf{X}](\lambda \mathbf{r}) \hat{\rho}(\mathbf{r}) d \mathbf{r} \\
& \left.+\int \lambda^{5-d} \mathbf{a}_{\alpha}[\rho, \mathbf{X}](\lambda \mathbf{r}) \cdot \hat{\mathbf{X}}(\mathbf{r}) d \mathbf{r}\right\} \\
& \times \Psi_{\alpha}^{\lambda}[\rho, \mathbf{X}]\left(\mathbf{r}_{1}, \ldots, \mathbf{r}_{N}\right)=\lambda^{2} E_{\alpha} \Psi_{\alpha}^{\lambda}[\rho, \mathbf{X}]\left(\mathbf{r}_{1}, \ldots, \mathbf{r}_{N}\right) .
\end{aligned}
$$

On the other hand, since $\Psi_{\alpha \lambda}\left[\rho_{\lambda}, \mathbf{X}_{\lambda}\right]$ is defined as the minimizing wave function in Eq. (7), $\Psi_{\alpha \lambda}\left[\rho_{\lambda}, \mathbf{X}_{\lambda}\right]$ obeys the following equation:

$$
\begin{aligned}
& \left\{\hat{T}+\alpha \lambda \hat{W}+\int v_{\alpha \lambda}\left(\rho_{\lambda}, \mathbf{X}_{\lambda}\right)(\mathbf{r}) \hat{\rho}(\mathbf{r}) d \mathbf{r}\right. \\
& \left.\quad+\int \mathbf{a}_{\alpha \lambda}\left(\rho_{\lambda}, \mathbf{X}_{\lambda}\right)(\mathbf{r}) \cdot \hat{\mathbf{X}}(\mathbf{r}) d \mathbf{r}\right\} \\
& \quad \times \Psi_{\alpha \lambda}\left[\rho_{\lambda}, \mathbf{X}_{\lambda}\right]\left(\mathbf{r}_{1}, \ldots, \mathbf{r}_{N}\right)=E_{\alpha \lambda} \Psi_{\alpha \lambda}\left[\rho_{\lambda}, \mathbf{X}_{\lambda}\right]\left(\mathbf{r}_{1}, \ldots, \mathbf{r}_{N}\right),
\end{aligned}
$$

where $E_{\alpha \lambda}, v_{\alpha \lambda}\left[\rho_{\lambda}, \mathbf{X}_{\lambda}\right](\mathbf{r})$ and $\mathbf{a}_{\alpha \lambda}\left[\rho_{\lambda}, \mathbf{X}_{\lambda}\right](\mathbf{r})$ are the Lagrange multipliers that correspond to constraints on $\Psi_{\alpha \lambda}\left[\rho_{\lambda}, \mathbf{X}_{\lambda}\right]$, that is, $\Psi_{\alpha \lambda}\left[\rho_{\lambda}, \mathbf{X}_{\lambda}\right]$ is normalized to unity and yields $\rho_{\lambda}(\mathbf{r})$ and $\mathbf{X}_{\lambda}(\mathbf{r})$. Here we consider the solution of Eq. (A4). The solution and potentials of Eq. (A4) should be determined in a self-consistent way. Namely, the potentials $v_{\alpha \lambda}\left[\rho_{\lambda}, \mathbf{X}_{\lambda}\right](\mathbf{r})$ and $\mathbf{a}_{\alpha \lambda}\left[\rho_{\lambda}, \mathbf{X}_{\lambda}\right](\mathbf{r})$ are determined by requiring that the solution $\Psi_{\alpha \lambda}\left[\rho_{\lambda}, \mathbf{X}_{\lambda}\right]$ is normalized and yields both $\rho_{\lambda}(\mathbf{r})$ and $\mathbf{X}_{\lambda}(\mathbf{r})$. If we choose the potentials as $\lambda^{2} v_{\alpha}[\rho, \mathbf{X}](\lambda \mathbf{r})$ and $\lambda^{5-d} \mathbf{a}_{\alpha}[\rho, \mathbf{X}](\lambda \mathbf{r})$, then the corresponding solution is immediately given by $\Psi_{\alpha}^{\lambda}[\rho, \mathbf{X}]$ from Eq. (A3). As shown in Eqs. (5) and (6), $\Psi_{\alpha}^{\lambda}[\rho, \mathbf{X}]$ is normalized and yields both $\rho_{\lambda}(\mathbf{r})$ and $\mathbf{X}_{\lambda}(\mathbf{r})$. Therefore, the solution of Eq. (A4), that is, $\Psi_{\alpha \lambda}\left[\rho_{\lambda}, \mathbf{X}_{\lambda}\right]$, can be equal to $\Psi_{\alpha}^{\lambda}[\rho, \mathbf{X}]$.

\section{APPENDIX B: PROOF THAT EQ. (17) IS REDUCED TO THE VIRIAL THEORUM IN THE LIMIT OF $\lambda=1$}

In this appendix, we show that Eq. (17) is reduced to the virial theorem in the limit of $\lambda=1$. By substituting $\lambda=1$ into Eq. (17) and using Eq. (2), we have

$$
\left.\frac{d E_{c}\left[\rho_{\lambda}, \mathbf{X}_{\lambda}\right]}{d \lambda}\right|_{\lambda=1}=T[\rho, \mathbf{X}]-T_{s}[\rho, \mathbf{X}]+E_{c}[\rho, \mathbf{X}]
$$

where $T[\rho, \mathbf{X}]$ is defined as $\langle\Psi[\rho, \mathbf{X}]|\hat{T}| \Psi[\rho, \mathbf{X}]\rangle$. On the other hand, the left-hand side of Eq. (B1) can be rewritten by

$$
\begin{aligned}
\left.\frac{d E_{c}\left[\rho_{\lambda}, \mathbf{X}_{\lambda}\right]}{d \lambda}\right|_{\lambda=1}= & {\left[\int \frac{\partial \rho_{\lambda}(\mathbf{r})}{\partial \lambda} \frac{\delta E_{c}\left[\rho_{\lambda}, \mathbf{X}_{\lambda}\right]}{\delta \rho_{\lambda}(\mathbf{r})} d \mathbf{r}\right.} \\
& \left.+\int \frac{\partial \mathbf{X}_{\lambda}(\mathbf{r})}{\partial \lambda} \cdot \frac{\delta E_{c}\left[\rho_{\lambda}, \mathbf{X}_{\lambda}\right]}{\delta \mathbf{X}_{\lambda}(\mathbf{r})} d \mathbf{r}\right]_{\lambda=1}
\end{aligned}
$$


If we assume that $\mathbf{X}_{\lambda}(\mathbf{r})=\lambda^{d} \mathbf{X}(\lambda \mathbf{r})$ and neglect integrals over an infinitely distant surface, then we get the following relation:

$$
\begin{aligned}
\left.\frac{d E_{c}\left[\rho_{\lambda}, \mathbf{X}_{\lambda}\right]}{d \lambda}\right|_{\lambda=1}= & -\int \rho(\mathbf{r}) \mathbf{r} \cdot \nabla\left[\frac{\delta E_{c}[\rho, \mathbf{X}]}{\delta \rho(\mathbf{r})}\right] d \mathbf{r} \\
& -\int \mathbf{X}(\mathbf{r}) \cdot\left[(\mathbf{r} \cdot \nabla-d+3) \frac{\delta E_{c}[\rho, \mathbf{X}]}{\delta \mathbf{X}(\mathbf{r})}\right] d \mathbf{r}
\end{aligned}
$$

Substituting Eq. (B3) into Eq. (B1), we obtain the virial theorem [10]:

$$
\begin{aligned}
& T[\rho, \mathbf{X}]-T_{s}[\rho, \mathbf{X}]+E_{c}[\rho, \mathbf{X}] \\
&=-\int \rho(\mathbf{r}) \mathbf{r} \cdot \nabla\left[\frac{\delta E_{c}[\rho, \mathbf{X}]}{\delta \rho(\mathbf{r})}\right] d \mathbf{r} \\
&-\int \mathbf{X}(\mathbf{r}) \cdot\left[(\mathbf{r} \cdot \nabla-d+3) \frac{\delta E_{c}[\rho, \mathbf{X}]}{\delta \mathbf{X}(\mathbf{r})}\right] d \mathbf{r} .
\end{aligned}
$$

[1] G. Vignale and M. Rasolt, Phys. Rev. Lett. 59, 2360 (1987).

[2] G. Vignale and M. Rasolt, Phys. Rev. B 37, 10685 (1988).

[3] M. Higuchi and A. Hasegawa, J. Phys. Soc. Jpn. 66, 149 (1997).

[4] M. Higuchi and A. Hasegawa, J. Phys. Soc. Jpn. 67, 2037 (1998).

[5] H. Ebert, M. Battocletti, and E. K. U. Gross, Europhys. Lett. 40, 545 (1997).

[6] S. Sharma, S. Pittalis, S. Kurth, S. Shallcross, J. K. Dewhurst, and E. K. U. Gross, Phys. Rev. B 76, 100401 (R) (2007).

[7] H. Saarikoski, E. Räsänen, S. Siljamäki, A. Harju, M. J. Puska, and R. M. Nieminen, Phys. Rev. B 67, 205327 (2003).

[8] S. Pittalis, S. Kurth, N. Helbig, and E. K. U. Gross, Phys. Rev. A 74, 062511 (2006).

[9] M. Higuchi and K. Higuchi, Phys. Rev. B 65, 195122 (2002).

[10] K. Higuchi and M. Higuchi, Phys. Rev. B 71, 035116 (2005).

[11] J. P. Perdew and Y. Wang, Phys. Rev. B 33, 8800 (1986).

[12] J. P. Perdew, in Electronic Structure of Solids, 91, edited by P. Ziesche and H. Eschrig (Akademie Verlag, Berlin, 1991), p. 11.

[13] J. P. Perdew, K. Burke, and M. Ernzerhof, Phys. Rev. Lett. 77, 3865 (1996).

[14] J. P. Perdew, A. Ruzsinszky, G. I. Csonka, O. A. Vydrov, G. E. Scuseria, L. A. Constantin, X. Zhou, and K. Burke, Phys. Rev. Lett. 100, 136406 (2008); 102, 039902(E) (2009).

[15] V. N. Staroverov, G. E. Scuseria, J. Tao, and J. P. Perdew, Phys. Rev. B 69, 075102 (2004).

[16] S. Liu and R. G. Parr, Phys. Rev. A 53, 2211 (1996).

[17] S. Liu, Á. Nagy, and R. G. Parr, Phys. Rev. A 59, 1131 (1999).

[18] Á. Nagy, S. B. Liu, and R. G. Parr, Phys. Rev. A 59, 3349 (1999).

[19] E. Bene and Á. Nagy, Chem. Phys. Lett. 324, 475 (2000).

[20] S. B. Liu, F. De Proft, Á. Nagy, and R. G. Parr, Adv. Quantum Chem. 36, 77 (1999).

[21] P. W. Ayers, J. B. Lucks, and R. G. Parr, Acta Phys. Chim. Debrecina 34-35, 223 (2002).

[22] P. Hohenberg and W. Kohn, Phys. Rev. 136, B864 (1964).

[23] W. Kohn and L. J. Sham, Phys. Rev. 140, A1133 (1965).
[24] M. Levy, Int. J. Quantum Chem. S23, 617 (1989).

[25] M. Levy and J. P. Perdew, Phys. Rev. A 32, 2010 (1985).

[26] A. Görling and M. Levy, Phys. Rev. A 45, 1509 (1992).

[27] Á. Nagy, Phys. Rev. A 47, 2715 (1993).

[28] R. G. Parr, S. Liu, A. A. Kugler, and Á. Nagy, Phys. Rev. A 52, 969 (1995).

[29] S. Erhard and E. K. U. Gross, Phys. Rev. A 53, R5 (1996).

[30] S. Liu, Phys. Rev. A 54, 1328 (1996).

[31] K. Higuchi and M. Higuchi, Phys. Rev. B 74, 195122 (2006); 75, 159902(E) (2007).

[32] M. Higuchi and K. Higuchi, Phys. Rev. B 75, 195114 (2007).

[33] K. Higuchi and M. Higuchi, J. Phys.: Condens. Matter 19, 365216 (2007).

[34] Y. Takada and H. Goto, J. Phys.: Condens. Matter 10, 11315 (1998)

[35] M. Higuchi and K. Higuchi, Phys. Rev. B 69, 035113 (2004).

[36] K. Higuchi and M. Higuchi, Phys. Rev. B 69, 165118 (2004).

[37] K. Higuchi and M. Higuchi, Phys. Rev. A 79, 022113 (2009).

[38] Á. Nagy, S. Liu, and L. Bartolloti, J. Chem. Phys. 122, 134107 (2005).

[39] Á. Nagy, Int. J. Quantum Chem. 106, 1043 (2006).

[40] M. Levy, Phys. Rev. A 43, 4637 (1991).

[41] O. Gunnarsson and B. I. Lundqvist, Phys. Rev. B 13, 4274 (1976).

[42] C. Lee, W. Yang, and R. G. Parr, Phys. Rev. B 37, 785 (1988).

[43] P. Skudlarski and G. Vignale, Phys. Rev. B 47, 16647 (1993).

[44] M. Rasolt and F. Perrot, Phys. Rev. Lett. 69, 2563 (1992).

[45] M. Ferconi and G. Vignale, Phys. Rev. B 50, 14722 (1994).

[46] O. Heinonen, M. I. Lubin, and M. D. Johnson, Phys. Rev. Lett. 75, 4110 (1995).

[47] M. Koskinen, J. Kolehmainen, S. M. Reimann, J. Toivanen, and M. Manninen, Eur. Phys. J. D 9, 487 (1999).

[48] A. Harju, S. Siljamäki, and R. M. Nieminen, Phys. Rev. B 65, 075309 (2002). 